\title{
Major Depressive Disorder: Current Advances and Paradigm Shifts
}

\author{
Yong-Ku Kim $\bowtie$ \\ Department of Psychiatry, College of Medicine, Korea University, Seoul, Republic of Korea
}

Major depressive disorder (MDD) is a multi-etiological, multi-dimensional, heterogeneous disease with variable symptomatology and treatment efficacy. Although many biological factors have been considered as risk factors for depression, their causal links remain unclear. Because depression is more likely to be a syndrome caused by various risk factors rather than a single etiology, whether the various findings are risk factors for depression or results observed in depression is not always clear. ${ }^{1}$

Although work in this area has been inconclusive, many animal, postmortem, clinical, and genetic studies have produced results implicating at least three neurobiological systems in the pathogenesis of MDD: the monoamine system, hypothalamic-pituitary-adrenal axis, and neuroplasticity. Additionally, other biological factors, including inflammatory markers, neurophysiologic markers, and neuroimaging markers, might be associated with MDD. Current approaches such as gene-environment interactions and epigenetics have been used to identify potential biomarkers for MDD. Accumulating neuroimaging studies suggest potential biomarkers such as metabolic activity, structural, or functional connectivity within the limbic-cortical circuitries. Considering the different biological mechanisms of depressive subtypes (atypical, melancholic, psychotic, and so on), it would be helpful to understand the pathogenesis of each depressive disorder to more accurately predict an individual's response to a specific treatment for depression. ${ }^{2}$

MDD has been reformulated based on a combination of categorical and dimensional approaches in the DSM-5. However, MDD is still defined using a symptom-based classification, and its heterogeneity is of significant concern. Moreover, it is necessary to develop an etiology-based classification of

\footnotetext{
$\triangle$ Correspondence: Yong-Ku Kim, MD, PhD

Department of Psychiatry, College of Medicine, Korea University, Ansan Hospital, 123 Jeokgeum-ro, Danwon-gu, Ansan 15355, Republic of Korea Tel: +82-31-412-5140, Fax: +82-31-412-5132, E-mail: yongku@korea.edu

(a) This is an Open Access article distributed under the terms of the Creative Commons Attribution Non-Commercial License (https://creativecommons.org/licenses/bync/4.0) which permits unrestricted non-commercial use, distribution, and reproduction in any medium, provided the original work is properly cited.
}

MDD and to consider humanistic approaches together with potential cultural and social influences. ${ }^{3}$ The Research Domain Criteria (RDoC) project by NIMH has been suggested as an alternative research-related methodology, however, it does not provide routine framework conditions for clinical psychiatry. The $\mathrm{RDoC}$ makes researchers and clinicians think about genetics, brain circuits, and neurophysiological factors in the context of abnormal behavior; however, $\mathrm{RDoC}$ neglects the clinical reality of sickness versus wellness in the lives of patients. While it is possible that research about neurocircuits and genes may eventually refine categories and identify subgroups that have biological validity and predict treatment response, this seems most plausible when examined in the context of a particular diagnosis. ${ }^{4}$

Treatment-resistant depression (TRD) is another important new area in medical and psychological services. About $30 \%$ of patients with MDD do not show substantial clinical improvement to somatic or psychosocial treatment. Most studies for TRD have focused on the patients already classified as TRD. Such a post hoc experimental design considered only the consequences of having TRD, rather than the causal risk factors for it. Accordingly, data derived from such studies often do not allow a distinction to be made between cause and effect. Thus, a paradigm shift toward examining the risk for developing TRD in untreated depressed patients is required. To deal with this problem, untreated depressed patients should be enrolled in a study to identify biological markers for treatment resistance. Hence, before starting treatment, peripheral or central biological markers should be explored. Subsequent systematic administration of treatments with appropriate monitoring in patients can determine the risk for developing treatment resistance in untreated individuals. Such information provides a clue to improving the initial diagnosis and providing more effective treatment for TRD. ${ }^{5}$

The special issue aims to provide current advances and paradigm shifts in major depressive disorders. Evrensel and colleagues shed light on neuroinflammation and the gut-microbiota-brain axis in major depressive disorder via their in- 
teresting review. ${ }^{6}$ The multiple effects of chronic low-grade inflammation initiated by chronic stress and depression on the integrity of the brain's neural network contribute to the neurotoxicity of proinflammatory cytokines, the modulation of biogenic amine neurotransmitters, and the activation of the neurotoxic arm of the tryptophan-kynurenine pathway. Neuroinflammation and neurodegeneration also affect intermediary metabolism of brain glucose as a result of the dysfunction of insulin, and could be the prelude for dementia in some cases of chronic depression. In addition, microorganisms affect the brain via the immune system, neuroendocrine system, and vagus nerve. Nutrition, stress, and medication lead to dysbiosis by changing the microbiota composition. Probiotic bacteria have a potential to be used in depression treatment. Fecal microbiota transplantation would be a hopeful sign for future cases of TRD.

Shin and $\mathrm{Kim}^{7}$ raise a new concept of therapeutic issues in MDD. Ketamine has received a great deal of attention over the last 20 years due to the discovery that a single subanesthetic dose leads to a rapid antidepressant effect in individuals with TRD. The antidepressant effects of ketamine are mediated through its antagonism of NMDA receptors and subsequent downstream effects on the enhanced activation of process and proteins leading to synaptogenesis. Given that a growing number of providers have begun offering ketamine off-label for the treatment of psychiatric disorders, future research is urgently needed to better understand longterm risks, as well as evidence-based treatment regimens.

The personalized or precision medicine approach to MDD is a very active avenue of investigation. Perna and colleagues emphasize the concept and usefulness of the personalized psychiatry approach in the clinical practice of treating MDD. ${ }^{8}$ In clinical practice, assessment of the sociodemographic and clinical variables can be valuable for early identification of depressed individuals at high risk for poorer responses to antidepressants, who may require a particular setting and a higher intensity of care and monitoring. Recent advances in computational resources such as machine learning techniques, which are able to integrate multiple potential predictors such as individual/clinical variables, biomarkers, and genetic factors, may offer future reliable tools to guide personalized antidepressant choices for each patient with MDD.

Orsolini and colleagues comprehensively review the risk factors, neurobiological changes, and reconceptualization of suicidal behaviors in MDD. ${ }^{9}$ Suicide is a highly complex and multifaceted phenomenon in which a plethora of mechanisms could be variably implicated, particularly in MDD. Scientific evidence suggests the synergic role of genetics, exogenous and endogenous stressors (i.e., interpersonal, professional, financial, as well as psychiatric disorders), epigenetics, the hypothalamic-pituitary-adrenal-stress-response system, the involvement of the monoaminergic neurotransmitter systems (particularly serotonergic systems), the lipid profile, neuro-immunological biomarkers, the brain-derived neurotrophic factor, and other neuromodulators.

I wish to give my heartfelt thanks to all authors for their valuable time spent preparing the manuscripts. These authors are leading research scientists with knowledge and expertise in their respective fields. I hope this special issue will be helpful and provide insight for better understanding and treating MDD.

\section{ORCID iD}

Yong-Ku Kim https://orcid.org/0000-0001-5694-7840

\section{REFERENCES}

1. Jeon SW, Amidfar M, Kim YK. Bio-Psycho-Social Risk Factors for Depression. In: Kim YK, Editor. Major Depressive Disorder: Risk Factors, Characteristics and Treatment Options. New York: Nova Science Publisher, 2017, p.71-90.

2. Lee HY, Kim YK. Pathophysiology and Treatment Strategies for Different Types of Depression. In: Kim YK, Editor. Understanding Depression. Vol 1. Biomedical and Neurobiological Background. Germany: Springer Nature, 2018, p.167-176.

3. Park SC, Kim YK. Depression in DSM-5: Changes, Controversies, and Future Direction. In: Kim YK, Editor. Understanding Depression. Vol 2. Clinical Manifestations, Diagnosis and Treatment. Germany: Springer Nature, 2018, p.3-14.

4. Weinberger DR, Glick ID, Klein DF. Whither Research Domain Criteria (RDoC)? JAMA Psychiatry 2015;72:1161-1162.

5. Jeon SW, Amidfar M, Kim YK. Paradigm Shift in Study of TreatmentResistant Psychiatric Disorder. In: Kim YK, Editor. Treatment Resistance in Psychiatry. Germany: Springer Nature, 2019, p.25-32.

6. Evrensel A, Unsalver BO, Ceylan ME. Neuroinflammation, gut-brain axis and depression. Psychiatry Investig 2020;17:2-8.

7. Shin C, Kim YK. Ketamine in major depressive disorder: mechanisms and future perspectives. Psychiatry Investig 2020;17:181-192.

8. Perna G, Alciati A, Daccò S, Grassi M, Caldirola D. Personalized psychiatry and depression: the role of sociodemographic and clinical variables. Psychiatry Investig 2020;17:193-206.

9. Orsolini L, Latini R, Pompili M, Serafini G, Volpe U, Vellante F, et al. Understanding the complex of suicide in depression: from research to clinics. Psychiatry Investig 2020;17:207-221. 